\title{
Cultural Logic and Syntactic Productivity: Associated Posture Constructions in Lao
}

\author{
N. J. ENFIELD
}

Languages with verb serialization provide speakers with a choice between expressing complex events as tightly cohesive multi-predicate yet single-clause structures, or as looser joinings of separate clauses. Bruce (1988: 25) has argued that the choice to employ one or the other means to describe a particular event is made in a principled way, whereby serial constructions 'are not merely more convenient (shortened) paraphrases' of looser multi-clausal structures. According to Bruce (1988: 28), '[serial constructions] must relate only events which are somehow conceived as notably more commonly associated together in experience or those events which form a culturally important concatenation of events'.

Durie (1997: 322) discusses a number of examples, and himself makes the following generalization:

[T]he productivity of verb serialisation is constrained in such a way that a large variety of syntactically well-formed verb combinations will be rejected by native speakers as unacceptable/ungrammatical because they do not correspond to a recognisable event-type, either within the actual experience of speakers, or alternatively within the permitted patterns of verb serialisation within a language. This can [. . .] apply even where particular verbs involved in a combination are otherwise able to combine in productive patterns of serialisation.

The issues Durie raises are broad-ranging, and it is my aim here to address and explore some of the problems, namely, (a) how we can make sense of a param-

Support and assistance in this research from Tony Diller, Grant Evans, and Syban Khoukham is gratefully acknowledged. The fieldwork was conducted in Lak Paet, Sisavat, and Saphang Mo villages of Vientiane, Lao PDR, between Novernber 1997 and May 1998. Helpful comments from members of the audience at the 'Ethnosyntax'workshop, Australian Linguistic Institute, Brisbane, July 1998 resulted in improvements. Also helpful were comments on various drafts of this chapter from Avery Andrews, Nick Evans, Cliff Goddard, Chris Manning, and Katherine Travis. My transcription of Lao (Southwestern Tai, Laos) is based on IPA convention, except glottal stop /q/, palatal and velar nasals / $\tilde{n}, n g /$, low central vowel $/ a /$, mid front vowel $/ \hat{e} /$, mid central vowel $/ e /$, low front vowel $/ \hat{e} /$, low back vowel $/ \grave{o} /$, and high back unrounded vowel /ül. Tones are marked by superscript numerals: 0 : unstressed, atonal; 1: level /33/: 2: high rising 134/; 3: low rising $/ 213 / ; 4$ : high falling $151 / ; 5$ : low falling $/ 21 /$. Abbreviations in glosses are as follows: $1 / 2 / 3$ (1st/2nd/3rd person pronoun); ACHV (achievement marker); C.LNK (clause linker); NEG (negative); PFV (perfective); PL (plural); SG (singular). 
eter like 'ready-recognizability of an event', which is a cultural notion more than it is a 'natural' or purely 'logical' one; $(b)$ how we can understand the notion of 'acceptability/grammaticality' given this view (and I remain sceptical about Durie's confident assertion of a culture-independent 'syntactic well-formedness' in these cases); and ( $c$ ) what kinds of grammatical and/or semantic effects related to this can be observed. I explore these points in turn, examining the last with reference to data from Lao.

I argue that event typicality is a cultural phenomenon, which can be accounted for and described in terms of cultural representations, typifications which are carried, assumed-to-be-carried, and assumed-to-be-assumed-to-be-carried by all members of a given group (Enfield 2000). For a complex event to be typical is for it to have currency, i.e. existing status as a cultural representation, readily available to speakers, in the public conceptual catalogue. Event typicality impacts directly upon the productive assembly of serial verb (and other) constructions, as well as the interpretation of the semantics of verb serialization, and of syntactic constructions in general.

This chapter is structured as follows. In Section 10.1, I outline the notion of 'culture' which I assume in subsequent discussion of grammar, pragmatics, and semantics. The important idea is the existence of conventions, which act as premisses in 'cultural logic' for interpreting utterances (and for judging them as 'grammatical' or not). Section 10.2 discusses the notion of event, and the relevance of this notion to the semantics and pragmatics of verb serialization. In Section 10.3, I turn to the examination of associated posture serial verb constructions in Lao, which provide a useful range of perspectives from which to focus in more detail on the observations made by Bruce and Durie, above. Section 10.4 provides a summary discussion of findings, and Section 10.5 concludes.

\subsection{CULTURAL LOGIC}

Linguistic studies which aim to address ethnographic issues must assume some view of just how facts of a cultural nature can be worked into the understanding of linguistic structure and usage. This section presents my own position on the nature of cultural conventions, and their role in the semantics and pragmatics of grammar.'

\subsubsection{Language and culture}

What we refer to as language and culture can be viewed as part of a mass of conceptual categories which are shared, assumed-to-be-shared, and assumed-to-

\footnotetext{
it The intention of this section is to describe as simply and briefly as possible my position on culture, and in the interest of space and clarity, I cannot make extensive reference to literature. The background is covered in detail in Enfield 2000.
} 
be-assumed-to-be-shared (D'Andrade 1987: 113), and which are formed, aligned, and manipulated via systematic deployment and/or interpretation of signs (in the broadest semiotic sense). This mass of ideas is not necessarily to be regarded as a coherent unitary system, but it does contain conceptual systems, with systematic relationships among categories. Beliefs and theories about 'what people do', 'what people think', 'what people say', 'what happens' in the world and why, are represented in this vast semiotic nebula. Therein, we encounter the common ideas which are to be found in the semiotics of symbols, cultural practices, and linguistic structures (Holland and Quinn 1987; Strauss and Quinn 1997).

I assume there are private representations, structured in the individual's body, mind, and brain. These are cognitive or sensorimotor in nature, and may take many forms (Enfield 2000: $37 \mathrm{ff}$.). Despite opposition by some to the notion of 'representations' (Foley 1997: 89), I find it necessary to recognize some kind of individualspecific internal abstractions (cf. Langacker 1994: 26-7), since individuals are clearly able to independently transport and access ideas which serve as premisses for cultural logic. Private representations may be internally manipulated by the individual (e.g. they may be recalled, or abstracted upon in various ways), and may include sensorimotor images of how certain practices are performed (e.g. techniques of tool use, posture), sense and emotional impressions, and propositional representations such as the theories, schemas, and norms abstracted from experience of the world (i.e. what people (do not) do, what can happen, what is good, what is bad, and so on).

Private representations are internally formed by individuals on the basis of 'artefactual' material that signifies meanings (i.e. 'mediating structure' such as sounds, Hutchins and Hazlehurst 1995: 56, 64). This includes all kinds of experiential input, from (either performed or observed) instances of complex and structured motor coordination (such as dancing or cooking), to audible phonetic material signifying linguistic expressions. By attending to similar artefactual structure, people jointly construct similar private representations. Prolonged and/or repetitive joint focus on certain mediating artefactual structure (along with mutual regulation, via cooperative discussion, correction, comparison, and internal review) allows us to achieve effectively identical private representations, via ongoing maintenance of conceptual alignment or convergence. ${ }^{2}$ Crucially, private representations are not literally 'passed' on, but are mediated by signs in use, and must be separately constructed by individuals in each case, based on the clues and 'instructions' that mediating structure provides (cf. Reddy 1979).

Thus, speakers effectively share conceptual/embodied private representations, and may therefore assume common carriage of these representations, across cultural/linguistic communities of arbitrary size (from two people to all people;

\footnotetext{
${ }^{2}$ Hockett's term intercalibration is appropriate here, too (Hockett 1987: 106-7, 157-8; cf. Lee 1996: 227-8). Unfortunately, space limitations do not allow a more detailed discussion of convention (cf. Lewis 1969; Clark 1996a, 1996b).
} 
Clark 1996a, 1996b). Knowledge is thus 'socially distributed' (Schutz 1946, 1970; Salomon 1993; Hutchins 1995). When carriers of a certain private representation know or assume that each other carries the private representation, and also know or assume that they themselves are similarly known or assumed by the other(s) to do so, then the private representation also becomes a cultural representation (Enfield 2000: 45), or, better, a cultural premiss. It then takes on a crucial role in cultural logic, as a default premiss for working out what is going on in social interaction (see below). Belonging to a certain carrier group means not having to overtly introduce the relevant cultural representation, since its presence is cooperatively enforced. It also means that in many cases one cannot deny that one carries it, or that one is a member of the said carrier group. Culture (but not necessarily 'a culture') can be defined in this way.

\subsubsection{Use of cultural premisses in interpretation of scenes and events}

Cultural premisses give us a guide to what happens in the world, i.e. what can be, and/or is more or less likely to be, the case. The fact that these ideas can be shared, and be known to be shared, means that human groups can utilize common guiding heuristics in interpreting the world in common ways (Levinson 1995: 240; Clark $1996 \mathrm{a}$ : ch. 4). Where certain cultural representations have a high level of activatedness and sharedness within a certain group, then individuals' interpretations of certain scenes/events are more likely to converge. Consider Fig. 10.1.

Corresponding to Fig. 10.1 $a$ is a cultural representation associated with situations in which a particular posture indicates acquiescence to discipline, as in school or the military, for which a description jùùn ${ }^{3} k{ }^{3} g^{3}$, literally 'stand straight', is readily selected by Lao speakers. For a Lao, the symbolism of the posture illustrated is unambiguous, and the activity of 'standing to attention' is not unfamiliar for modern Lao people (as for example would be expected in certain situations

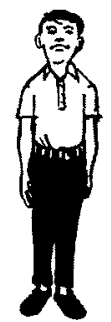

(a)

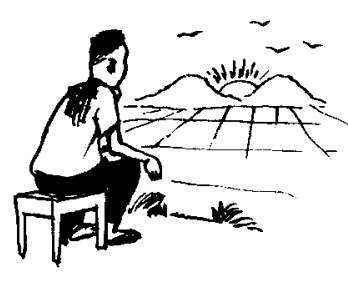

(b)

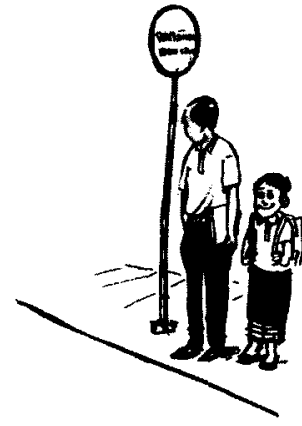

(c)

FIG. 10.1 
at school). Note that Australian informants generally do not describe Fig. 10.1a with the equivalent term standing to attention, apparently since the idea of standing to attention is more contextually restricted in Australia (i.e. the feet would not be apart, for example, and the subject would be more obviously uniformed). On the other hand, Figs. $10.1 b$ and $10.1 c$ do not obviously correspond to salient cultural representations for many Lao speakers. The range of responses to Fig. $10.1 b$ is revealing. Some informants were not familiar at all with the conventional illustration of the setting/rising sun, or even the conventional graphic perspective depicting receding fields and distant hills. Instead, two informants guessed that the person in the picture was making a fire. ${ }^{3}$ Others said that the person was 'watching over their fields', or 'appreciating nature'. The point here is that while Lao people of course appreciate the beauty of the setting/rising sun, there is no salient cultural representation for Lao speakers corresponding to English watching the sunset/sunrise. The practice of specifically putting aside time to behold the sight is not categorized as 'something that people do'.

Fig. 10.1 $c$ also elicited unusual and inconsistent responses from Lao speakers, again due to the lack of a highly salient or current corresponding cultural representation. The artist had been asked to sketch $k h o n^{2}$ jùùn ${ }^{3}$ thaa ${ }^{5}$ lot $^{1}-m e \hat{e} \hat{e}^{2}$ [person stand wait bus] 'people standing, waiting for a bus'. The sketch he produced corresponds to a bus-stop stereotype from a developed country like Australia, where bus stops are signposted places by curbed streets where people wait for buses. But in Laos, few places where people get on buses are signposted in this way. People usually wait at familiar corners or roadheads, where they simply flag a passing bus down. While some people did describe Fig. $10.1 \mathrm{c}$ as 'people (standing) waiting for a bus', others described the scene as 'people (standing) waiting to cross the road', and one even described it as 'people having their height measured'. This final interpretation is revealing in terms of the heuristic value of cultural representations as inferential premisses, and the way in which the relative salience and/or assumed sharedness of cultural representations comes into play in interpreting scenes. The speaker in question saw nothing familiar in the illustration corresponding to a 'busstop' scenario, despite the artist's intention. She instead recognized the banded pole as a measuring rule, since she had a pre-existing private representation of this already available (presumably due to her personal experience, perhaps in a community health centre, or the like). And she could assume that this idea/experience was likely to be shared by her interlocutors. For her, it was the idea of the heightmeasuring scenario that enabled the best solution to the 'coordination problem' (Schelling 1960; Lewis 1969) of interpreting Fig. 10.1c. The process of finding that solution involved selection from her catalogue of available cultural representations. Evidently for this speaker, an elderly rural woman, the idea of the modern bus-stop scenario, as illustrated, was not available. In other words, she did not

\footnotetext{
${ }^{3}$ This is a good example of a supposedly 'iconic' sign--the sun setting/rising on the horizonwhich is actually not iconic at all, but conventionalized and symbolic; cf. Noble and Davidson 1996: ch. 3 for discussion of the putative iconicity of symbols.
} 
carry (or at least did not assume to be mutually carried) the idea which the artist had assumed would have currency among those who would see his illustrations. It is these principles of reliance on salient and/or current cultural representations which are argued below to play a crucial role in the production and interpretation of syntactic constructions.

\subsubsection{How cultural representations feed into cultural logic}

Available cultural representations are employed as premisses in cultural logic, the system of reasoning behind our explanations of events, and attributions of intentions to agents and other event participants. ${ }^{4}$ Consider the different interpretations a Lao and an Australian would have of a diner drinking water at the last stage of a meal. A Lao normally takes this as a sure sign that the diner has completely finished eating, and indeed this reading regularly compels people to act, for example by clearing away plates, or by (rhetorically) urging one's guest not to stop eating so soon. An Australian, however, would not 'logically' attribute this meaning to the act of drinking water. The difference is that Lao people have a cultural representation in the form of a meal 'script' which reserves the drinking of water for the moment of having finished eating. According to the Lao 'script', people do not drink water while they are eating a meal. Of course, Lao people can drink water during a meal, and indeed some do. But it is conventionally assumed that they do not, and that the default reading of the act of drinking water towards the end of a meal is that one has finished eating. Exceptions are recognized as such. Australians, on the other hand, have no particular cultural representation or script concerning the timing and/or overlap of water and food consumption.

Another example concerning food and dining is the Lao practice of eating together from communal plates and bowls, rather than serving out meal portions individually. Many foreigners in Laos find this 'impractical'. One Australian, for example, wondered aloud why the Lao do not at least each have their own bowl for soup, since transport of hot soup in the spoon is especially prone to spillage. This suggestion to her seemed 'logical'. But for the Lao, any argument that the communal arrangement is 'impractical' is outweighed by local cultural conventions that prioritize personal proximity, camaraderie, and solidarity in day-to-day family-internal activities. Separate personal bowls, even for soup, would create uncomfortable social distance in the normally informal eating situation, since maintenance of proximity and sharing is culturally important among Lao coinhabitants. The Lao communal eating arrangement provides important symbolic manifestation of this cultural preoccupation.

\footnotetext{
${ }^{4}$ On cultural logic, see Enfield 2000; cf. Schelling 1960, Garfinkel 1967; Hutchins 1980; Gumperz 1982; Brown and Levinson 1987 (see in particular pp. 8, 64-5, 84-90); Wierzbicka 1991; Levinson 1995 (anther papers in Goody 1995); Clark 1996a.
} 


\subsubsection{How cultural representations and cultural logic feed into 'grammaticality'}

An important area of syntactic research where cultural representations come into play is in the context of eliciting grammaticality judgements as a methodology in syntactic description (cf. Diller and Khanittanan, this volume). One parameter against which people judge the acceptability of utterances is that of event typicality, which is determined, as described above, by cultural conventions. Consider the putative ungrammaticality of the following example (Kay 1996: 112):

\section{(1) *Sybil had Sidney fall off the couch.}

The matrix causative verb have requires that its complement verb have an agentive subject. In this case, however, according to Kay, 'the complement verb [fall] doesn't allow its subject to express an agent' (Kay 1996: 112), so there is a semantic clash and the sentence is rejected. But then Kay shows that (1) is acceptable after all, 'if, for example, Sybil is thought of as a stage director and Sidney's descent represents not real falling but an actor's deliberate simulation' (Kay 1996: 112). So why is (1) deemed ungrammatical? Why would this be 'not real falling'? On what basis are we to make use of this conditional grammaticality in syntactic description? In the right context, (1) is a completely normal utterance. I argue that this falling is indeed 'real falling'. It is merely atypical. Fall does not entail nonagentivity, it only implies it (because 'People "don't" fall on purpose').

Adjustment of the context of (1) by invoking the 'theatre direction' context also relies on cultural representations, albeit marked, or less salient, ones. There may come a point where context-adjustment becomes conceptually too demanding. ${ }^{5}$ For reasons of what is 'logically possible' - what people would or would not ('ever', let alone 'normally') imagine possible - the utterance may not be accepted, or, indeed, may not even be easily interpreted. Here we find the rejection of marked combinations in verb serialization that Durie describes, for example in an expression like *She took a fish (to the market) and bought it (Durie 1997: 326; cf. Sebba 1987: 60). However, even this utterance may be fine given the right context-say, some ritual purchase. ${ }^{6}$ Such a case would not be an example of 'not real buying', but, again, merely atypical buying. It remains unclear to me on what basis one would reject the string on 'logical' grounds, while maintaining the notion of 'syntactic well-formedness' (Durie 1997).

\footnotetext{
${ }^{5}$ Speakers often use the 'heuristics' of cultural typicality to bypass having to resolve interpretations of difficult-to-process structures-hence, for example, the persistent failure of subjects to correctly interpret the meaning of 'verbal illusions' such as No head injury is too trivial to ignore (which 'really' means 'All head injuries can be ignored, the most trivial'; Wason and Reich 1979).

${ }^{6}$ Thanks to Nick Evans for pointing this out.

Another important factor in acceptability of such constructions is the culture-specific willingness of informants to indulge in hypothesis about possible situations, especially when these seem unlikely or unconfirmable (cf. Luria 1976: $108 \mathrm{ff}$.).
} 


\subsubsection{Culture and syntax: discussion}

I have argued that cultural premisses, which provide communities with massive shared backgrounds of common heuristics for convergent cultural logic, come into constant play in all kinds of interaction, and especially in the online interpretation of linguistic utterance meaning. Below, I assume this view of culture and cultural logic, which indeed I see as providing an account for the workings of language itself, whether that concerns the encoded semantics of lexical and grammatical signs, or the context-and assumption-based processes of pragmatic inference and interpretation that pervade our everyday use of language (Grice 1975; Levinson 2000 ). Both the semantic and the pragmatic in language are at all times cultural.

More specifically, the knowledge required for interpretation and production of syntactic strings is not just knowledge of the structural mechanisms available for combination of meaningful linguistic units, but also, and just as importantly, the possible, and most normal or unmarked, co-occurrences of morphemes in these structures. The claim that cultural typifications can have significant effects on syntactic productivity, and indeed processability, makes ethnosyntax an important field of study even for nativist approaches to syntax. The study of syntax must pay more attention to the productivity of syntactic structures and the combinatorial properties of specific structural elements. ${ }^{8}$

In sum, cultural premisses define what is typical, feeding into cultural logic, guiding and/or constraining the assembly and interpretation of syntactic constructions in context. In Section 10.3, below, we focus on a concrete example, examining the role of event typicality in culture-related constraints on the productivity and interpretation of a type of verb serialization in Lao. But we must first address the notion of event, among other aspects of the semantics of verb serialization.

\subsection{DESCRIPTION OF COMPLEX EVENTS}

The event is a fundamental concept in grammatical description and in semantics (cf. Grace 1987; Langacker 1987; Talmy 2000; inter alia). Most simply, there has been an event if we can say that something has happened. ${ }^{9}$ Almost all, if not all, languages contain a formally distinct word class which prototypically denotes events (i.e. the 'verb' class), and many simple events are described by simple single-verb clauses. Of course, no event in the world is literally simple, and no event is literally bounded, but we can and do abstract away the uniqueness and complexity of given segments of reality, and we find (or imagine) bounded events within the flux of impressions. In describing events, we strip out a great amount of detail,

\footnotetext{
${ }^{8}$ Recent traditions in 'Construction Grammar' (Goldberg 1995; Kay and Fillmore 1999, inter alia) focus on issues of syntactic productivity.

${ }^{9}$ I assume that the description of events cannot get any more conceptually basic than the simple categories 'happen' and 'do'; Wierzbicka (1996: 50).
} 
and categorize unlike events in like ways. What is of primary interest here is how events are conceptualized, not how they are, since it is the former that is embodied in linguistic semantics. Linguistic signs describe, but do not necessarily reflect, the way of the world.

Important work has been done on the notion of the conceptual event (Grace 1987; Pawley 1987; Foley 1997: 37), the idea that events may be conceptualized as unitary and contained, regardless of whether they are obviously made up of component-events, and/or whether their linguistic expression is formally simple or complex. In cases of complex formal structures, this single-event status is often diagnosed with reference to intonational chunking, and other indices of conceptual closure (Chafe 1994: ch. 5). Such conceptually unitary events may be either simple or complex in terms of their (both conceptual and formal) internal structure. I assume that at least some event descriptions are so simple we would seldom conceptually dismantle them. Consider Mary opened her eyes and John died at 9 o'clock. Other event descriptions like John washed the car and Mary sold her house, although conceptually unitary, are at the same time unproblematically and naturally recognized by speakers as consisting of more than one sub-event.

\subsubsection{Description of complex events: alternative fashions of speaking}

The means speakers employ in describing complex events are often determined by convention, as evidenced by the ubiquity of idiomatic metonymy (Lakoff 1987: 78-9; Pawley 1987: 339). Compare American English go to the bathroom with Lao khaw ${ }^{5}$ hò̀ng $^{5}$-nam ${ }^{4}$ [enter room-water] 'enter the bathroom'. Both refer to essentially the same conceptual event which includes, among other things, the subevents of 'going' and 'entering'. It so happens that English speakers conventionally refer to the whole event by explicit reference to just the 'going' component, while Lao speakers explicitly mention just the component of 'entering'. In both cases, one sub-event ('going' or 'entering') stands metonymically for the whole complex event. This sub-event metonymy indeed applies productively in the two languages-while in English one also 'goes to' both hospital and prison, in Lao, one 'enters' these institutions. Another example of different metonymies for much the same complex event is English wash the dishes versus Lao laang $^{4}$ thuaj ${ }^{5}$ [wash bowl] 'wash the bowls' - both of these conventionally refer to a category of complex event which involves washing of not only dishes and bowls, but other vessels and utensils, too.

Many events which speakers need to describe of course do not neatly match categories that are already encoded by simple verbs or stock idioms, and one primary function of grammar is to provide speakers with ways to express novel detail in the description of events. Speakers employ a range of resources, including novel predicate-argument combinations, adverbial constructions, adjuncts, particles, strategies of verb subordination and coordination, verb compounding, and verb serialization. We now consider this last strategy. 


\subsubsection{Construction of multi-component and multi-facet events via verb serialization}

Event descriptions employing verb serialization consist formally of multiple predicate-like elements, but the predications characterized by these complex expressions are conceptually unitary. This is an important and often definitive generalization made about serial verb constructions and similar multi-verb sequences which form structural (and especially intonational) chunks (Pawley 1987; Givón 1991; Lord 1993; Durie 1997). Indeed, it is from work on verb serialization that the very notion of conceptual event, discussed above, has emerged.

Events can be complex in at least two ways. First, a conceived event may consist of multiple separately discernible component-events, which can be imagined as separate, especially in terms of their temporal relationship, with one componentevent following another. Consider (2), a Lao serial construction in which a series of component-events are predicated, in iconic order:

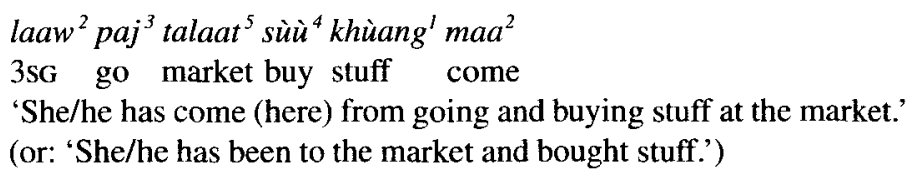

With a single intonation contour, (2) may be regarded as expressing a unitary conceptual event, while also consisting of easily discernible separate componentevents. Now, a clause linker lèk $a^{0}$ may be inserted between verb phrases, with the effect of explicitly breaking up those component-events into separated, temporally sequenced events, each then describing a separate conceptual event:

(2') laaw $^{2}$ paj $^{3}$ talaat $^{5}$ lèka $^{0}$ sùù $^{4}$ khùang $^{1} l e ̀ k a^{0}$ maa $^{2}$

3sG go market C.LNK buy stuff C.LNK come

'She/he went to the market, and then bought stuff, and then came (here).'

The effect in (2') is to separate the component-events into distinct events in their own right, but the basic 'real-world' scenarios described by $(2)$ and $(2$ '), respectively, remain the same. There are still three events which happen one after the other, namely, those designated by the verbs $p a j^{3}$ 'go', sùu' 'buy', and maa 'come'. The difference in description is one of conceptual closure--in (2) they are componentevents of a single conceptual event, while in $\left(2^{\prime}\right)$ they are each separate conceptual events in themselves.

Events which are complex in a second, separate sense, may consist of multiple event-facets, which, again, speakers are willing to recognize as constitutive of a complete event, but which in this case are not separable from other elements of the event, particularly not in terms of temporal precedence. Rather, event-facets are as if overlaid to form a more detailed and complete event description. Typical multifacet events include those which combine manner with direction of motion (He ran away, Mary cycled down the hill), or posture with action (She slept standing, John sat writing a letter). 
Consider the following example of a multi-facet event in Lao:

(3) laaw $w^{2}$ lèèn ${ }^{1}$ long $^{2} \quad$ maa $^{2}$

3sG run descend come

'She/he ran down (here).'

This directional serial verb construction predicates manner, path, and direction, in that order. These facets of the event are conceptually overlaid, and not separable in the same way as the sub-events of (2). Thus, we get a very different result when the clause linker lek $k a^{0}$ is inserted between verbs (cf. ( $\left.2^{\prime}\right)$, above):

(3') laaw ${ }^{2}$ lèèn lèka ${ }^{0}$ long $^{2} \quad l e ̀ k a^{0} m a a^{2}$

3sG run C.LNK descend C.LNK come

'She/he ran, and (then) came/went down, and (then) came.'

The scenarios described by (3) and ( $3^{\prime}$ ) are very different. In (3), there is one action, namely 'running', and predicated of that 'running' in addition is that it is downward in path, and towards the speaker (or some transposed 'speaker' locus) in direction. But in ( $\left.3^{\prime}\right)$, there are three separate events, and there is no entailment that the motion predicated by lèèn' 'run' is downward or towards the speaker, nor is the downward motion predicated by long ${ }^{2}$ 'descend' (or the towards-speaker motion predicated by $\mathrm{maa}^{2}$ 'come') necessarily done in the manner of 'running'.

In Section 10.3 below, we examine serial constructions of this second type-i.e. in which the verbs in combination describe overlaid facets of a single happening.

\subsubsection{How cultural logic constrains the assembly of complex event descriptions}

In the context of the quote given at the start of this chapter, Durie (1997) notes the unacceptability of certain combinations of verbs in serial constructions as "puzzling'. Consider the following examples (cited in Durie 1997: 329):

(4) White Hmong (Jarkey 1991: 169-70)

a. nws dhia tshov qeej

3sG dance blow bamboo.pipes

'He dances playing the pipes.'

b. *nws dhia mloog nkauj

3sg dance listen song

(He dances and listens to music.)

(5) Alamblak (Bruce 1988: 29)

a. miyt ritm muh-hambray-an-m tree insects climb-search.for-1s-3PL

'I climbed the tree looking for insects.'

b. *miyt guñm muh-hëti-an-m

tree stars climb-see-1s-3PL

(I climbed the tree and saw the stars.) 
According to Jarkey, while ( $4 a$ ) is a natural, unitary event (whenever the qeej pipes are played, the performer dances along with the music), dancing and listening (as in (4b) ) are 'normally viewed as distinct events' (Durie 1997: 329). ${ }^{10}$ Similarly for Alamblak, $(5 a)$ is a 'readily recognisable event-type', while $(5 b)$ is not (Bruce 1988: 29). Durie (1997: 327) rightly points out that the criteria of 'stereotypical schema for event-types' are very likely to be culture-specific, and that there is thus expected to be some variation across cultures of what 'the normal view' constraining expressions of complex events entails.

In the context of the range of issues raised so far, let us now consider some examples of 'associated posture' serial verb constructions in Lao.

\subsection{ASSOCIATED POSTURE CONSTRUCTIONS IN LAO}

Lao associated posture constructions are multi-verb sequences in which a posture verb (typically one of $\operatorname{nang}^{1}$ 'sit', jùùn ${ }^{3}$ 'stand', or $n \grave{o ̀ n}{ }^{2}$ 'lie', which I will refer to hereafter as the basic postures) directly precedes a verb denoting some action. ${ }^{11}$ The posture verb and the action verb are tightly bound elements of a single verb phrase core, with a single intonation contour. These are verb compounds - there is no obvious reason to consider either the posture verb or the activity verb as head of the predicate to the exclusion of the other-which provide translation equivalents to adverbial or depictive expressions in English (e.g. John studied [lying down]). The following sections present cases of syntactic and semantic/pragmatic variation associated with particular combinations of posture and activity in these grammatical constructions. Relevant grammatical details are elucidated where necessary en route.

I investigated this problem from two angles. First, I asked speakers to comment on constructed sentences involving various 'posture'+ 'activity' combinations. Second, I conducted picture-description tasks using a set of sixteen simple sketches, depicting a variety of activities (see Figs. 10.1-10.9). The sketches manipulate two parameters: posture (sitting vs. standing vs. lying), and activity (varying with respect to degrees of typicality in any or all of the basic postures-for example, among the activities depicted, both reading and watching television are normal in all postures, but slightly marked standing, while riding a motorcycle and playing the lanaat ${ }^{4}$ are almost never done standing or lying down). Other pictures included posture + activity combinations known to have salient cultural/linguistic representations (i.e. they are very typical, such as the combination of 'lying' and 'sleeping', and/or have unique labels, such as 'standing to attention'; cf. discussion in s. 10.1.2,

\footnotetext{
${ }^{10}$ Actually, this seems to be a case, as seen with the example from Kay (1996) above, of the binary nature of grammaticality judgements giving misleading results. My own field investigations with Hmong speakers in Vientiane (in 1999) reveal that $(4 b)$ is odd because it is so atypical as an event-but it is nevertheless not unacceptable.

1 For the purpose of this discussion, I ignore subtle differences in meaning between English sit, stand, lie, and Lao expressions glossed herein as 'sit', 'stand', and 'lie'.
} 
above). In some cases, the results of standard elicitation based on extracting grammaticality judgements in response to constructed strings were significantly different from those based on elicitation of posture descriptions.

\subsubsection{An 'activity'typically done lying down: 'sleeping'}

That Lao people typically sleep lying down is not due to anything unique about Lao culture:

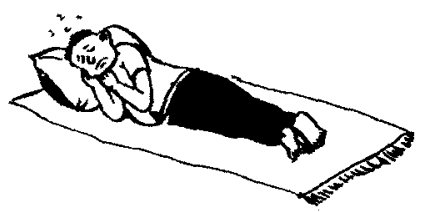

Fig. 10.2.

The fact of all humans' sleeping lying down is determined, presumably, by 'common phylogenetic inheritance and common terrestrial fate' (Levinson 1997: 281). But the idea that 'people typically sleep lying down' is nevertheless a cultural one, in the sense of 'cultural' described in Section 10.1.1 above-i.e. everyone carries an idea that 'sleeping is done lying down', everyone knows that everyone carries this idea, and everyone knows they themselves are known to carry it. The conceptual combination of 'lying' and 'sleeping' is not merely logical, but culturally logical.

The Lao verbs nòòn 'lie' and lap ${ }^{2}$ 'be asleep' combine in describing, most idiomatically, an event in which someone has gone to sleep (e.g. as in Fig. 10. 2):

(6) laaw $w^{2}$ nò̀n ${ }^{2} l a p^{2}$

3SG lie be.asleep

'She/he is asleep.'

Example (6) entails that the subject is asleep. It is also common to use nòn ${ }^{2}$ 'lie' alone as a metonymic expression for 'go to bed/go to sleep', but to do so does not entail that the person has actually gone to sleep, merely that they are in a lying posture.

Now, it is also possible to refer to other (indeed atypical) cases of sleeping, using lap $^{2}$ 'be.asleep' in combination with nang 'sit' or jùùn ${ }^{3}$ 'stand':

(7) laaw $^{2}$ nang $^{1}$ lap $^{2}$

3sG sit be.asleep

'She/he slept/is asleep sitting.'

(8) $\quad l a a w^{2}$ jùùn ${ }^{3} l a p^{2}$

3sG stand be.asleep

'She/he slept/is asleep standing.' 
Clearly, these would only be felicitous in describing unusual circumstances, such as a crowded bus trip, where the sleepy traveller cannot lie down, nor perhaps even sit. While these require more unusual non-default contexts, they nevertheless correspond to readily recognizable situations with an appeal to the shared 'script' of arduous and cramped travelling. In elicitation sessions, examples like (7) and (8) are questioned initially, but are readily accepted as natural once a satisfying context is provided (cf. discussion of example (1) in s. 10.1, above).

The cultural typicality of the combination nòòn ${ }^{2} a p^{2}$ 'lie.down'+'be.asleep', illustrated in (6), has significant consequences in terms of morphosyntactic behaviour. The combination has properties of a resultative construction, one of the few V1-V2 combinations in Lao which allows insertion of the negative marker bò ${ }^{I}$ between the initial and resultative verbs, signifying failure to achieve the result (V2) despite completion of the precipitating action (V1):

(9) $l a a w^{2}$ nòòn ${ }^{2} b \grave{o} \grave{o}^{l} l a p^{2}$

3sG lie NEG be.asleep

'She/he couldn't/can't get to sleep.' (cf. example (6), above)

The syntactic behaviour of this culturally logical combination is, in contrast, not shared by the atypical basic posture compound combinations nang ${ }^{1}$ lap $^{2}$ [sit be.asleep] 'sleep sitting' and jùùn ${ }^{3} l a p^{2}$ [stand be.asleep] 'sleep standing' (cf. examples (7), (8), above):

$$
\begin{aligned}
& \text { ??laaw }{ }^{2} \text { nangl }^{\prime} \text { bòò } \text { lap }^{2} \\
& \text { 3sG sit NEG be.asleep }
\end{aligned}
$$

(Sitting, she/he couldn't get to sleep, i.e. She/he sat, but couldn't sleep.)

$$
\begin{aligned}
& \text { ??laaw }{ }^{2} \text { jùùn }{ }^{3} \text { bò̀̀ }{ }^{l} \text { lap } \\
& \text { 3sG } \quad \text { stand NEG be.asleep }
\end{aligned}
$$

(Standing, she/he couldn't get to sleep, i.e. She/he stood, but couldn't sleep.)

Of course, the ideas behind (10) and (11) can be expressed, but this is done with looser, less prosodically integrated grammatical structures, such as the following (suggested by informants as preferred ways of expressing (10) and (11)):

$$
\begin{array}{lll}
\text { laaw }^{2} & \text { nang }^{l} \text { maa }^{2} \text { bòo } \grave{o}^{l} l a p^{2} \quad \text { sut }^{2} \quad \text { thaang }^{2} \\
\text { 3SG sit come NEG be.asleep extremity way }
\end{array}
$$$$
\text { 'She/he sat coming here, and didn't sleep the whole journey.' }
$$

$$
\begin{aligned}
& \text { laaw } w^{2} \text { jùùn }{ }^{3} j u u^{\prime} \quad b \grave{o}{ }^{l} d a j^{0} \quad l a p^{2} \quad l e e j^{2} \\
& \text { 3sG stand be.at NEG ACHV be.asleep at.all } \\
& \text { 'She/he was standing, and didn't get to sleep at all.' }
\end{aligned}
$$

Both Bruce (1988) and Durie (1997) have similarly observed that less readily recognizable event-types are less likely to be expressible in the tight multipredicate structures which conventionally express unitary 'conceptual events'. 
10.3.2. Two activities not unusual in any posture: 'reading' and 'watching television'

The activities of reading and watching television are both typically done either sitting or lying down, and are also not uncommonly done standing up. In neither activity is sitting or lying posture any more typical than the other. But in both cases, to do the activity standing is at least marked with respect to the other two. Since watching television and reading are sedentary pastimes, demanding essentially only mental and visual(/aural) attention, and requiring no mobility, one is naturally inclined to rest the body while engaging in these activities. Standing is not a resting posture, but essentially a posture of readiness either to move away or to adopt a more restful posture having just stopped moving. ${ }^{12}$

It is common for people to walk up to a television, turn it on to check what is on, and remain standing, pending a decision to continue watching (in which case one sits or lies down), or walk away and do something else. This is a common scenario, personally embodied and conceptually stored as a cultural typification for members of television-watching human groups, as 'something that people do'. Similarly, to stand and read a book is not particularly unusual, but is marked in that it would only be done when one is perhaps briefly scanning a text, or standing for a particular reason (e.g. as a teacher may stand in front of a class while reading aloud).

Thus, it is not surprising that Lao informants find associated posture constructions involving 'read' and 'watch television' perfectly acceptable with any of the basic postures:

(14) a. nò̀̀n ${ }^{2}$ beng ${ }^{1}$ thoo ${ }^{2}$ lathat ${ }^{l}$

lie look television

'watch television lying down'

b. nang' beng' thoo ${ }^{2}$ lathat ${ }^{\prime}$

sit look television

'watch television sitting down'

c. jùùn ${ }^{3}$ beng $^{\prime}$ thoo'lathat ${ }^{\prime}$

stand look television

'watch television standing up'

Two interesting effects may be noted here. First, due to the markedness of the standing posture in $(14 c)$, informants are apparently compelled-without prompting-to provide further information explaining the more marked circumstances, that is, to activate in their interlocutor's mind the less salient cultural premiss which feeds cultural logic for coherent interpretation of the event. A typical comment was: 'The sentence in $(14 c)$ is fine-for example, you might be just checking to see whether there is anything interesting on.'

\footnotetext{
${ }^{12}$ There are also practical and/or cultural reasons to be standing, e.g. while trying to get a view, or
} while teaching a class, but these do not normally apply while watching television. 
Second, when presented with illustrations of possible combinations of the three postures 'sitting', 'standing', and 'lying' with the two activities 'watching television' and 'reading a book', in almost every case speakers used an associated posture construction to explicitly specify the relevant posture in their initial description of each sketch:

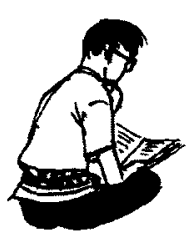

(a)

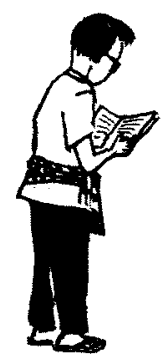

(b)

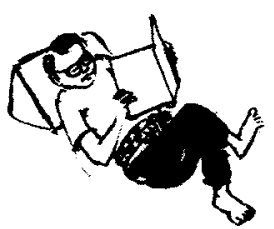

$(c)$

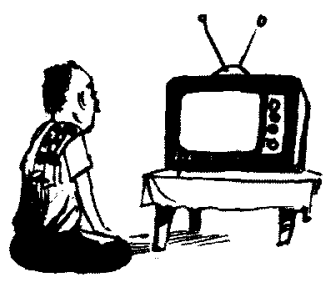

(d)

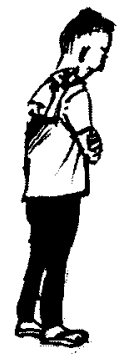

$(e)$
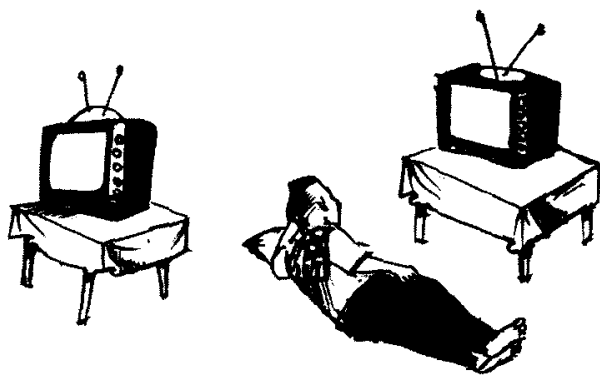

$(f)$

FIG. 10.3.

In these cases, no posture is more typical than the others, and therefore no posture is privileged as a default. The point about defaults is that they do not need to be specified, which is a handy thing in language, since it allows us to economize by leaving out details which we can assume will be 'automatically' understood (Grice 1975: 45; Clark 1996a: 70; Levinson 2000: 37). Thus, for example, if shown a scene in which John gives Mary a scarf, in describing it you are unlikely to bother mentioning the fact that he used his hand in doing so. On the other hand, if in the scene John uses his foot in giving her the scarf, then one would be highly likely to mention this in a description. ${ }^{13}$ In the cases of watching television and reading

${ }^{13}$ This is borne out by results of recent comparative field research on 'event typicality' by members of the Language and Cognition Group at the Max Planck Institute for Psycholinguistics in Nijmegen. 
a book (Fig. 10.3), speakers are virtually obliged to explicitly specify the posture adopted in the given scene, since no single posture is likely to be understood by default.

\subsubsection{Three activities typically done sitting}

\subsubsection{Playing the lanaat ${ }^{4}$}

The lanaat ${ }^{4}$ is a traditional Lao instrument, a kind of concave xylophone, played on the ground in a seated position, as in Fig. 10.4. To know what the word lanaat ${ }^{4}$ means, you need to know, among a number of cultural representations about the instrument, that it is played in a sitting (on the ground) posture. The verb most often used for 'playing' the lanaat is $\mathrm{til}^{3}$ 'hit, beat'. The following constructed examples were supplied to informants for judgement of their acceptability:

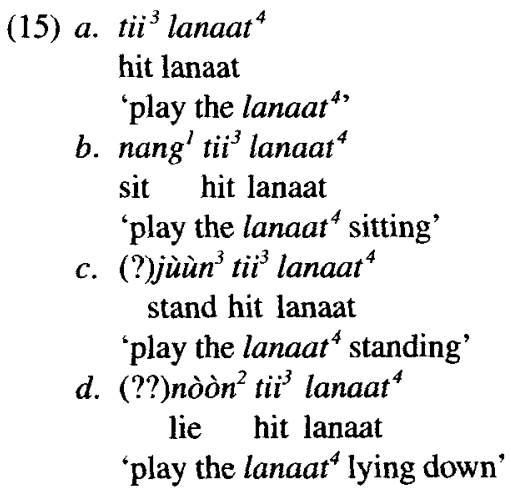

Informants judged examples (15a) and (15b) as perfectly natural, according directly with cultural representations associated with the lanaat ${ }^{4}$. Despite there being no specification of posture, speakers assume that the subject of $(15 a)$ is seated in the traditional manner. Example (15c), on the other hand, was met with

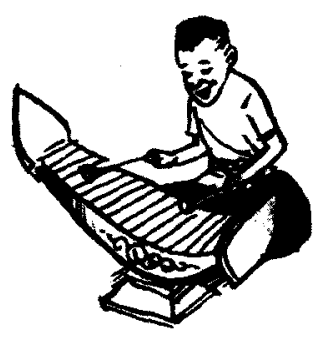

Fig. 10.4 
some hesitation, but in most cases judged to be acceptable, usually with a comment to the effect that 'one could play a lanaat ${ }^{4}$ standing up, if it were on a table, say, but people don't do that'. Again, speakers are compelled-consistently, and without prompting - to offer additional information 'explaining' pragmatically marked situations. In interpreting (15c), the informant pauses to search in his catalogue of cultural premisses for a sensible interpretation of the unexpected combination. Finally, informants are much less prepared to accept example (15d), in the spirit of the quote from Durie (1997: 322) at the beginning of this chapter. A number of informants actually rejected this string when it was presented out of context. One commented, 'It doesn't make sense-you can't play the lanaat ${ }^{4}$ lying down.'

These findings on the event combinations in $(15 a-d)$ were derived by presenting constructed sentences to informants and asking whether they are possible utterances. Very different results emerge from a methodology which begins with extensional data-i.e. illustrations of real scenes - and asks the informant for a description. The three illustrations of 'playing the lanaat ${ }^{4}$ ' were Figs. 10.4 and 10.5. In the picture-labelling task, the informant no longer has to struggle to imagine the situation actually taking place. Strikingly, in describing Fig. $10.5 b$, every informant spontaneously produced the structure in $(15 d)$, a string which most informants in elicitation sessions found difficult to accept, and which some had rejected outright. Fig. $10.5 b$ gives the informant the very context in which the atypical combination of 'lying down' and 'playing the lanaat ${ }^{4}$ ' is normal, namely an informal situation in which a musician is practising or playing for fun in the privacy of his own home, and not as a normal (public) performance. Given only the sentence $(15 d)$, many speakers simply didn't think of a fitting scenario, apparently too fixated on the typical scene of a performer seated in the traditional way (Fig. 4 , above). Thus, event typicality apparently restricts the imagination, the ability to think laterally, in this case with respect to possible scenes involving the activity

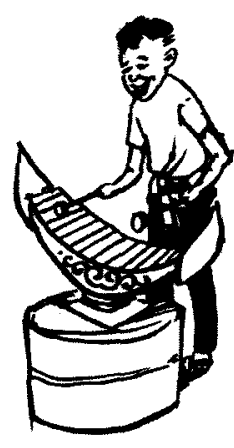

(a)

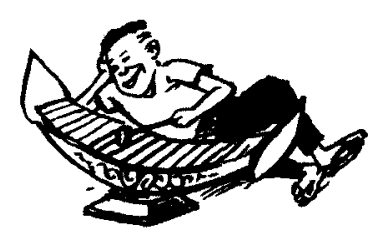

(b)

FIG. 10.5 
of 'playing the lanaat". Given the sentence (15d), once the typical scenario of 'playing the lanaat ${ }^{4}$ has become activated in the speaker's mind, the highly salient solution-i.e. that the agent is sitting -is arrived at, and this results in the 'locking off' of further interpretation. Thus, it is hard to imagine someone 'lying down and playing the lanaat ${ }^{4}$. However, seeing the illustration in Fig. 10.5b, informants are not forced to search their own conceptual 'store' for a licensing cultural representation (in competition with much more salient combinations). The use of certain morphosyntactic means for description of the scene in a way which had seemed unacceptable out of context becomes completely natural, in fact optimal.

Another issue arising from exposure to the three illustrations of 'playing the lanaat ${ }^{4}$ (Figs. 10.4 and 10.5) is the very decision to employ the associated posture construction at all-i.e. to explicitly specify in the description the posture adopted. Recall that in s.10.3.2 above, in response to illustrations of 'watching television' and 'reading a book', speakers spontaneously used associated posture constructions for all three basic postures, due to lack of cultural premisses specifying a unique posture as default. By contrast, among scenes of 'playing the lanaat", Fig. 10.4 (the stereotypical scene, with the musician seated) almost never elicited an associated posture construction. It was not specified that the person playing the lanaat ${ }^{4}$ was seated-informants just said, 'He's playing the lanaat ${ }^{4}$.' The musician's sitting posture in Fig. 10.4 is literally unremarkable. That speakers much more readily omit mention of posture in describing this particular 'posture'+'activity' combination suggests that the factors determining speakers' choice as to whether a certain grammatical construction will be used at all may also be essentially cultural.

An important result emerges here concerning a somewhat hazy line in the semantics of verb serialization-namely, the distinction between compositional and idiomatic semantics. The meaning of a syntactically complex expression may or may not be a neat sum of its constituent semantic parts. In some cases the meaning of the construction may need to be specified as a lexical entry in itself. Of interest here is the verb $t i i^{3}$, which literally means 'hit' or 'beat', and may also mean 'play' (a musical instrument). By metonymy, the 'play' reading of $t i^{3}$ ' $h i t$ ' involves not just 'hitting', but orderly hitting, resulting in something we recognize as being the proper sound to emerge from a lanaat ${ }^{4}$. But one may still use the expression $t^{3}{ }^{3}$ lanaat ${ }^{4}$, literally 'hit the lanaat ${ }^{4}$, to describe an event in which someone simply strikes the body of the instrument, perhaps with a stick, in a completely unmusical way. This reading would involve purely compositional semantics. Even so, the expression $t_{i i^{3}}$ lanaat ${ }^{4}$ out of context is very likely to receive a 'play' reading for $t i^{3}$, not simply 'hit'. Here we observe a window of slippage in 'verb serialization' with respect to the lexicalization/syntax distinction. One informant who rejected (15d), which specifies playing the lanaat ${ }^{4}$ in the atypical lying posture, found the expression perfectly acceptable given the context of a child lying down aimlessly hitting the instrument (i.e. not 'playing' it), in which case $t i i^{3}$ was interpreted as 'hit' (and the meaning of the overall expression was then purely compositional- 
'lying down and hitting a lanaat ${ }^{4}$ ). The semantic construal of the verb $t i i^{3}$ in the syntactic combination $t i i^{3}$ lanaat ${ }^{4}$ as either 'hit' or 'play' can be pushed one way or the other by the particular posture specified in a serial combination. Ultimately, then, the construal of the whole complex syntactic expression can be directly contingent upon cultural representations.

\subsubsection{Fang ${ }^{2}$-thêêt ${ }^{4}$ 'listening-to-sermon'}

A second example of an activity typically done sitting is that of $\mathrm{fang}^{2}$-thêe $t^{4}$ 'listening to (a) sermon'. In Laos, worshippers may visit the temple on $\operatorname{van}^{2}-\sin ^{3}$ 'holy days', and partake in a form of worship which involves, among other things, ritually listening to the sermon (referred to as thêe $t^{4}$ ) of Buddhist monks. Like any cultural practice, fang $^{2}$-thêe $t^{4}$ is to some extent embodied through personal participation, and to some extent emicized by its conceptualization linked to semiotic material (at the very least involving people's usual ways of talking about the practice). One performs the practice of fang ${ }^{2}$-thêe $t^{4}$ in order to hêt $t^{I} b u n^{3}$ 'make merit'. One enters

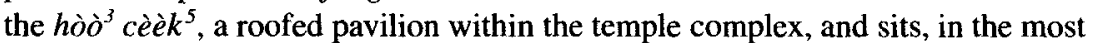
polite or respectful sitting posture, namely phap ${ }^{I}$-phèè ${ }^{4}$ (i.e. on the floor, with both legs tucked under and back and to the same side), and with palms pressed together in front of one's chest, in the gesture known as nop ${ }^{l}$ (see Fig. 10.6).

Monks performing the sermon (usually chanted in formulaic Pali) are seated higher than the congregation. These various stipulations are familiar to Lao speakers as cultural representations surrounding the practice of fang $^{2}$-thêe $t^{4}$, regardless of whether one actually engages, or indeed has ever engaged, in the practice. That is, if you know what fang ${ }^{2}-$ thêe $t^{4}$ is, as an established Lao Buddhist practice associated with the ritual accrual of religious merit, you know that one does it sitting down. The following example shows that specification of the sitting posture in an associated posture construction is optional (as for 'playing the lanaat ${ }^{4},(15 a, b)$, above):

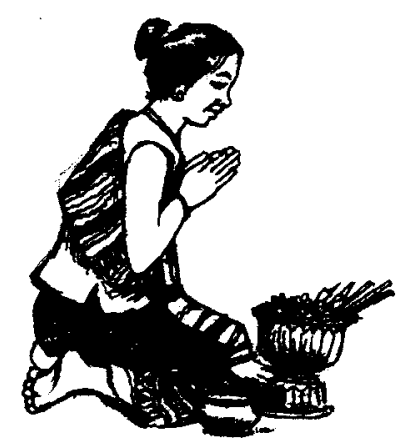

FIG. 10.6 


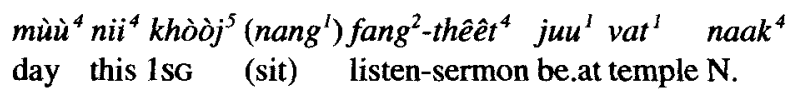

The meaning of the combination fang ${ }^{2}$-thêe $t^{4}$ in this example is not simply combinatorial, i.e. not semantically merely the sum of fang ${ }^{2}$ 'listen' and thêêt ${ }^{4}$ 'sermon'. While only the 'listening' and the 'sermon' are explicitly mentioned, with them are imported the complex of culturally defined specifications of the whole event, including the fact that the subject is intentionally and legitimately participating in a religious ritual performance (with the belief that merit will accrue as a result), and adopting the standard and legitimate postures, clothing, gestures, and proxemics defined by relevant cultural representations.

Rather different interpretations emerge from associated posture constructions combining fang ${ }^{2}$-thêe $t^{4}$ with non-sitting postures:

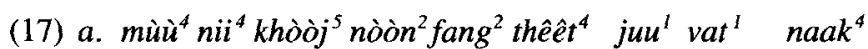
day this $1 \mathrm{SG}$ lie listen sermon be.at temple $\mathrm{N}$.

'Today I lay down and listened to a sermon at Nak Temple.'

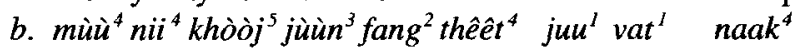

day this $1 \mathrm{sG}$ stand listen sermon be.at temple $\mathrm{N}$.

'Today I stood and listened to a sermon at Nak Temple.'

In both (17a) and (17b), the semantic combination of fang ${ }^{2}$ 'listen' and thêe $t^{4}$ 'sermon' is purely compositional, in that there is no entailment or even implication of anything more than 'listening' and 'sermon'. Crucially, the subject in (17a, $b$ ) is understood by informants not to be participating in the religious performance known as fang ${ }^{2}$-thêe $t^{4}$, even though fang ${ }^{2}$ the $\hat{e} t^{4}$ [listen sermon] is literally predicated. If no posture of the subject is predicated, the subject of the predicate fang ${ }^{2}$-thê $t^{4}$ is assumed by default to be seated (as illustrated in Fig. 10.6), and explicit mention of sitting posture is also perfectly felicitous (if superfluous). But if a non-sitting posture is explicitly predicated, then due to the incompatibility of a non-sitting posture with cultural premisses of the $\mathrm{fang}^{2}$-thêêt ${ }^{4}$ scenario, the listener knows that the subject cannot be properly performing the ritual. The 'logical' conclusion is that the person is merely listening to the sound of the sermon, as is literally predicated, perhaps while waiting in the temple grounds for a friend. Informants agree that the non-seated subject of (17a) and (17b) cannot be properly 'listening-to-sermon' with the intention to make merit, nor would he be in the hò ${ }^{3}$ cè̀e $k^{5}$ itself, where the practice is rightly done.

\subsubsection{Riding a motorcycle}

Motorcycles are designed to accommodate the human body in what is essentially a sitting position (although not a prototypical one), i.e. one in which the weight of the body is mainly on the posterior, so that the hands and legs are free and mobile enough to operate the vehicle (see Fig. 10.7). The unmarked predication of 'rid- 
ing a motorcycle' strongly implies this sitting posture. Of the following examples, (18a), with no posture specified, is consistently judged by informants to be most natural:

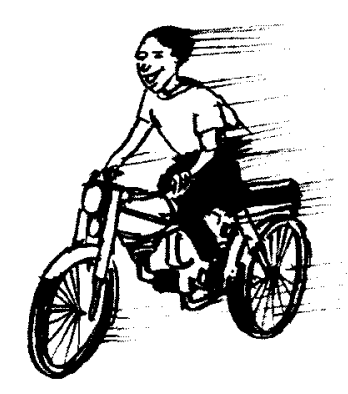

Fig. 10.7.

(18) a. $k$ hii $^{1} \operatorname{lot}^{I}-\mathrm{cak}^{2}$

ride motorcycle

'ride a motorcycle'

b. nang $^{I}$ khii $^{l}$ lot $^{l}-$ cak $^{2}$

sit ride motorcycle

'ride a motorcycle sitting down'

c. $n \grave{o ̀ n}{ }^{2} k h i i^{I}$ lot $^{1}-$ cak $^{2}$

lie ride motorcycle

'ride a motorcycle lying down'

d. jùùn ${ }^{3}$ khii' $^{1}$ lot $^{1}$-cak ${ }^{2}$

stand ride motorcycle

'ride a motorcycle standing'

Overt specification of sitting posture in $(18 b)$ was generally considered by informants to be superfluous (more so than in the cases of 'playing the lanaat' ${ }^{4}$ and ' $\left.f a n g^{2}-t h e \hat{e} t^{4 '}\right)$, while the specifications of lying and standing posture in $(18 c, d)$ produced similar results to examples $(15 c, d)$ and $(17 a, b)$ discussed above. That is, informants were at first non-accepting, due to a lack of 'ready-recognizability' of these complex events. But they were quickly accepting once fitting contexts were activated.

Other informants were presented with an illustration of someone standing on a moving motorcycle (Fig. 10.8). Some responded with (18d), while others omitted mention of the verb $k h i i$ ' 'ride' altogether, giving jùùn' 'stand' as a sole main verb, not as an associated posture verb:

(19) jùùn ${ }^{3}$ juu $u^{\prime}$ theng ${ }^{2}$ lot $^{\prime}-c a k^{2}$

stand be.at atop motorcycle

'(He's) standing on a motorcycle.' 


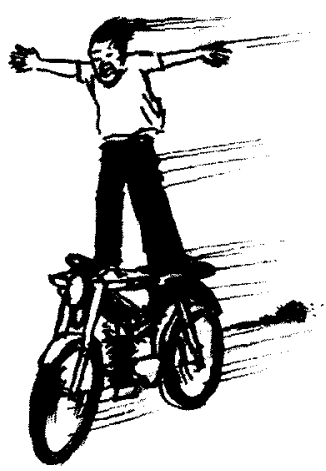

Fig. 10.8 .

Others mentioned $k h i i^{l}$ 'ride' alone as a main verb, but focused on the fact that the rider's hands were not controlling the bike, as in the following:

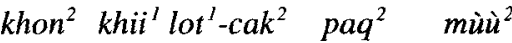

$$
\begin{aligned}
& \text { person ride motorcycle abandon hand } \\
& \text { '(It's) a person riding a motorcycle with (his) hands free.' }
\end{aligned}
$$

Speakers found the combination of 'lying down' and 'riding a motorcycle' somewhat more difficult to describe in a number of ways (Fig. 10.9). It seems that when nòòn 'lying down', one's weight must be supported along the length of one's body. The area of available supporting upper surface of a motorcycle is too restricted for this, and there was some debate as to whether it was possible to nòon ${ }^{2}$ 'lie down' on a motorcycle at all. A number of informants described Fig. 10.9 with an associated posture construction using the postural verb mêêp ${ }^{5}$ 'to have one's weight forward on one's chest', as when one falls forward onto the ground:

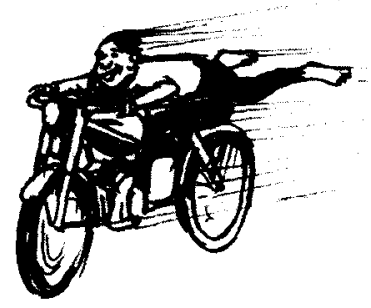

FIG. 10.9. 
As described in other cases above-for example with respect to the combination of 'watching television' and 'standing', Section 10.3.2, above-informants presented with the more marked circumstances (in Figs. 10.8 and 10.9) felt independently compelled to offer explanations as to what the circumstances must be. For instance, the most common remark about Fig. 10.8 was that it 'must be a stunt show'. The stunt-show cultural representation is less salient among all of those that involve riding a motorcycle, but it is nevertheless one that provides a solution to the coordination problem of interpreting the given complex event, and it has sufficient currency to be evoked in making sense, in this way, of what the illustrator must have intended. The fact that it is marked compels the speaker to activate the idea in the consciousness of his interlocutor, in order to ensure that his interlocutor is sharing the right premisses for collective convergence upon the appropriate culturally logical solution.

\subsection{DISCUSSION: FIVE FINDINGS}

This chapter has examined a number of aspects of event typicality and the semantic and grammatical productivity of verb serialization, with particular reference to data from Lao. This has been based on two complementary methods of investigation-first, constructing example sentences and asking for interpretations and judgements of their acceptability; second, using a picture-description task to elicit spontaneous linguistic descriptions. The results suggest five general observations.

First, the dependence on cultural logic for interpretation of semiotic ("mediating artefactual') structures, including grammatical and morphosyntactic material, entails personal search and retrieval of cultural representations which facilitate the best, most likely, and most 'logical' solutions. When the relevant cultural representations are assumed to be of lower salience and/or lower currency, speakers are compelled to overtly mention their markedness, in order to activate non-default cultural representations in the minds of interlocutors, ensuring convergent culturally logical solutions. This is the essence of Grice's maxim of Manner, or Levinson's 'M-heuristic', whereby 'what's said in an abnormal way isn't normal' (Levinson 2000: 38). What I add to this is to stress that 'what is normal' is culturally defined.

Second, the notion of grammaticality, crucial to standard methodology in grammatical description and analysis, makes constant and direct reference to the heuristics provided by cultural typifications, by which we assess the typicality or plausibility of situations predicated. Research methodology must be revised in this light, not only with respect to verb serialization, but in syntactic research in general (Diller and Khanittanan, this volume). With no satisfying context available, a given sentence may be rejected, and therefore considered by the analyst (or the speaker) 
to be out of the range of description, i.e. 'not part of the grammar'. Primed with the right context, however, speakers may spontaneously produce such 'unacceptable' utterances. The relevant parameter-event typicality or markedness of conceptual combination-is a cultural one.

Third, the status of possible combinations of conceptual sub-components in complex expressions as more or less 'normal' (as culturally defined) affects the accessibility of such combinations to certain productive morphosyntactic processes (e.g. insertion of various connectors or logical relators, marking of modal and aspectual distinctions, ellipsis, specific intonational properties, prosodic integration, etc.).

Fourth, construal of the semantics of verb serialization, which straddles a blurry line between true syntactic composition and conventionalized/idiomatic meaning (usually involving metonymy), is contingent upon cultural typifications, in that the overall meanings of serial verb constructions will be construed as pragmatically enriched where existing cultural representations encourage or license this, and as purely syntactic/combinatorial elsewhere.

Fifth, the choice as to whether or not to employ a certain syntactic construction at all is contingent upon cultural typifications, since the latter provide a measure of plausibility or markedness of the component sub-events of a conceptualized complex event. For example, in Lao, an associated posture construction will be used when the particular 'posture'+ 'activity' combination in a given scene is pragmatically marked, i.e. requires a less salient or current cultural representation for culturally logical interpretation. When one particular posture is uniquely typical in combination with a certain activity, the associated posture construction is less likely to be used at all, since the relevant posture, being the default, need not be explicitly mentioned.$^{14}$ Such constraints on whether a morphosyntactic device will be used at all can be regarded as properly part of the 'rules' of syntax, since these constraints are a crucial part of what it takes for speakers to string utterances together.

\subsection{CONCLUSION}

The idea that the fine cogs and springwheels of syntax might be guided and/or constrained by the culture of speakers is an intriguing one, self-evident to some, opaque to others, and strongly resistant to clear articulation by anyone. The problem has received little attention in syntactic research relative to other preoccupations, due in part to the descriptive and analytical difficulties of bringing ethnography into the equation. Speakers effortlessly navigate the most delicate yet robust intricacies of linguistic and cultural systems, while almost completely

\footnotetext{
${ }^{14}$ It has long been recognized that there is no need to mention what is already present by default. It is literally unmarked. This is the essence of Grice's second maxim of Quantity: 'Do not make your contribution more informative than is required' (Grice 1975: 45; cf. Levinson's 'I-heuristic', 2000: 37) What I am trying to stress here is that $(a)$ what is considered to be already 'automatically' understood by default is to a large extent culturally determined, and $(b)$ this directly affects syntax, since it determines when and/or whether a certain syntactic construction will be used at all.
} 
unconscious of the systematicity of these semiotic resources. However, the great difficulty of the task is no argument that ethnosyntactic description cannot or should not be undertaken. Despite the difficulties, and the dangers of traversing uncharted terrain, I submit that the unified description of grammar and culture from a broad perspective is a necessary and timely complement to the often narrow concerns of traditional syntactic research.

\section{REFERENCES}

Brown, Penelope, and Levinson, Stephen C. 1987. Politeness: Some Universals in Language Usage. Cambridge: Cambridge University Press.

BRuCE, Les. 1988. 'Serialization: from syntax to lexicon'. Studies in Language, 12.1: $19-49$.

Chafe, WAllace. 1994. Discourse, Consciousness, and Time: The Flow and Displacement of Consciousness in Speech and Writing. Chicago: Chicago University Press.

Clark, Herbert H. 1996a. Using Language. Cambridge: Cambridge University Press.

1996b. 'Communities, commonalities, and communication'. In Gumperz and Levinson 1996: 324-55.

D'ANDRADE, RoY D. 1987. 'A folk model of the mind'. In Holland and Quinn 1987: 11250.

DURIE, MARK. 1997. 'Grammatical structures in verb serialization'. In Alex Alsina, Joan Bresnan, and Peter Sells (eds.), Complex Predicates, Stanford, Calif.: CSLI Publications, 289-354.

ENFIELD, N. J. 2000. 'The theory of cultural logic: how individuals combine social intelligence with semiotics to create and maintain cultural meaning'. Cultural Dynamics, 12.1: 35-64.

Foley, William A. 1997. Anthropological Linguistics: An Introduction. London: Blackwell.

Garfinkel, HAROLD. 1967. Studies in Ethnomethodology. Englewood Cliffs, NJ: PrenticeHall.

Givón, TALMY. 1991. 'Serial verbs and the mental reality of "event": grammatical vs. cognitive packaging'. In Berndt Heine and Elizabeth Traugott (eds.), Approaches to Grammaticalization. Amsterdam: John Benjamins, 81-127.

GoldBerg, Adele. 1994. Constructions: A Construction Grammar Approach to Argument Structure. Chicago: University of Chicago Press.

GOODY, EsTHER N. (ed.). 1995. Social Intelligence and Interaction: Expressions and Implications of the Social Bias in Human Intelligence. Cambridge: Cambridge University Press.

Grace, GeORGE. 1987. The Linguistic Construction of Reality. London: Croom Helm.

Grice, H. P. 1975. 'Logic and conversation'. In Peter Cole and Jerry Morgan (eds.), Syntax and Semantics, iii: Speech Acts. New York: Academic Press, 41-59.

GuMPERZ, JoHN J. 1982. Discourse Strategies. Cambridge: Cambridge University Press.

-and Levinson, StePhen C. (eds.). 1996. Rethinking Linguistic Relativity. Cambridge: Cambridge University Press.

Hockett, Charles F. 1987. Refurbishing our Foundations: Elementary Linguistics from an Advanced Point of View. Amsterdam: John Benjamins. 
Holland, Dorothy, and Quinn, NaOmi (eds.). 1987. Cultural Models in Language and Thought. Cambridge: Cambridge University Press.

Hutchins, Edwin. 1980. Culture and Inference: A Trobriand Case Study. Cambridge, Mass.: Harvard University Press.

-1995. Cognition in the Wild. Cambridge, Mass.: MTT Press.

- and HAZLEHURST, BRIAN. 1995. 'How to invent a shared lexicon: the emergence of shared form-meaning mappings in interaction'. In Goody 1995: 53-67.

JARKEY, NerIDA. 1991. 'Serial verbs in White Hmong: a functional approach'. Doctoral dissertation, University of Sydney.

KAY, PAUL. 1996. 'Intra-speaker relativity'. In Gumperz and Levinson 1996: 97-114.

- and Fillmore, Charles. 1999. 'Grammatical constructions and linguistic generalizations: the What's $X$ Doing $Y$ ? construction'. Language, 75.1: 1-33.

LAKOFF, GeORGE. 1987. Women, Fire, and Dangerous Things: What Categories Reveal about the Mind. Chicago: University of Chicago Press.

LANGaCker, Ronald W. 1987. Foundations of Cognitive Grammar, i: Theoretical Prerequisites. Stanford, Calif.: Stanford University Press.

- 1994. 'Culture, cognition, and grammar'. In Martin Pütz (ed.), Language Contact and Language Conflict. Amsterdam: John Benjamins, 26-53.

Lee, Penny. 1996. The Whorf Theory Complex: A Critical Reconstruction. Amsterdam: Benjamins.

LEVINSON, STEPHEN C. 1995. 'Interactional biases in human thinking'. In Goody 1995: 221-60.

- 1997. 'From outer to inner space: linguistic categories in non-linguistic thinking' In Jan Nuyts and Eric Pederson (eds.), Language and Conceptualization. Cambridge: Cambridge University Press, 13-45.

2000. Presumptive Meanings: The Theory of Generalized Conversational Implicature. Cambridge, Mass.: MIT Press.

LewIS, David. 1969. Convention. Cambridge, Mass.: Harvard University Press.

LORD, CAROL. 1993. Historical Change in Serial Verb Constructions. Amsterdam: John Benjamins.

LuRIA, A. R. 1976. Cognitive Development: Its Cultural and Social Foundations. Cambridge, Mass.: Harvard University Press.

Noble, William, and Davidson, IAIN. 1996. Human Evolution, Language, and Mind: A Psychological and Archeological Inquiry. Cambridge: Cambridge University Press.

PAWLey, ANDrew. 1987. 'Encoding events in Kalam and English: different logics for reporting experience'. In Russell Tomlin (ed.), Coherence and Grounding in Discourse. Amsterdam: John Benjamins, 329-60.

REDdy, MICHAEL. 1979. 'The conduit metaphor: a case of frame conflict in our language about language'. In Andrew Ortony (ed.), Metaphor and Thought. Cambridge: Cambridge University Press, 284-324.

Salomon, Gavriel (ed.). 1993. Distributed Cognitions: Psychological and Educational Considerations. Cambridge: Cambridge University Press.

Schelling, Thomas C. 1960. The Strategy of Conflict. Cambridge, Mass.: Harvard University Press.

SCHUTZ, ALFRED. 1946. 'The well-informed citizen: an essay on the social distribution of knowledge'. Social Research, 13.4: 463-78. 
SCHUTZ, ALFRED. 1970. On Phenomenology and Social Relations. Chicago: University of Chicago Press.

SEBba, Mark. 1987. The Syntax of Serial Verbs. Amsterdam: John Benjamins.

Strauss, Claudia, and Quinn, Naomi. 1997. A Cognitive Theory of Cultural Meaning. Cambridge: Cambridge University Press.

TAlmy, LeONARD, 2000. Toward a Cognitive Semantics, 2 vols. Cambridge, Mass.: MIT Press.

WASON, P. C., and REICH, C. C. 1979. 'A verbal illusion'. Quarterly Journal of Experimental Psychology, 31: 591-8.

WierzBicka, ANNA. 1991. Cross-Cultural Pragmatics: The Semantics of Human Interaction. Berlin: Mouton de Gruyter.

_1996. Semantics: Primes and Universals. New York: Oxford University Press. 